\title{
The Fracture Toughness of Medium Density Fiberboard (MDF) Including the Effects of Fiber Bridging and Crack-Plane Interference
}

\author{
Noah Matsumoto and John A. Nairn \\ Wood Science \& Engineering, Oregon State University, Corvallis, OR, USA
}

\begin{abstract}
The fracture toughness of medium density fiberboard (MDF) as a function of crack length ( $R$ curve) was measured. Fracture toughness was determined from force-displacement and crack length data using a new energy analysis procedure that avoids the scatter of prior discrete analysis methods. Because crack lengths were difficult to observed, they were measured using digital image correlation (DIC). The $R$ curves for two different densities of MDF, two thicknesses, and for both in-plane and through-the-thickness cracks all increased linearly with crack length. The increase was interpreted as the development of a fiber-bridging process zone. Numerical modeling methods were used to determine the cohesive stress of the fiber-bridging zone.
\end{abstract}

Keywords: Fracture toughness; fiber bridging; crack interference; energy methods; wood; composite; cohesive stress; material point method

\section{Introduction}

Most fracture mechanics standards, such as ASTM E399 (2006), implicitly assume selfsimilar crack propagation, where "self-similar" means that two samples with different crack lengths differ only by their crack lengths. This assumption is violated in materials that develop process zones, such as fiber bridging in composites. In such materials, the initial crack will have no process zone, but a zone will develop during propagation. Thus, even if crack propagation is 
straight, the initial and final samples will differ both by their crack lengths and by their process zone size. A consequence of such non-self-similar crack growth is that common fracture standards cannot be used. Non-self-similar crack growth, however, does not invalidate fracture mechanics. It only means that alternative methods are needed for measuring fracture toughness.

This paper illustrates a variety of complications that arise when measuring the fracture toughness of composites by describing experiments on medium density fiberboard (MDF). MDF is a wood-based composite with fine wood fibers bound together by a small amount of polymeric adhesive (Bower, et al., 2006). Under mode I loading, MDF cracks generally propagate straight and perpendicular to the applied load. The cracks, however, are difficult to detect and clearly have a significant amount of material bridging the crack surfaces (Matsumoto and Nairn, 2007). As a result, the propagation is not self similar. An alternative to fracture mechanics standards, which still applies for non-self-similar crack growth, is to directly measure energy released during crack propagation. A common experimental protocol is to load until a small amount of crack growth and then unload and measure the area between the loading and unloading curves (Hashemi, et al., 1990). This method could not be used for MDF because the process zone interfered with the unloading step. We resolved all issues by using continuous loading experiments with simultaneous optical detection of the crack length. Analysis of the experiments resulted in measurement of a valid toughness for MDF. The implications for measuring fracture toughness of other composites are discussed.

Figure 1b illustrates four orthogonal crack directions in an MDF panel - LT, TL, ZL, and ZT. The first letter indicates the normal to the crack surface and the second letter denotes the crack propagation direction with $\mathrm{L}, \mathrm{T}$, and $\mathrm{Z}$ standing for in-plane (L)ongitudinal, in plane (T)ransverse, and the thickness (Z) directions, respectively (Bodig and Jayne, 1982). MDF 
panels are manufactured to be nominally isotropic for in-plane properties although the throughthe-thickness properties are different. We measured toughness in the above four crack orientations in the form of $R$ curves or toughness as a function of crack length. As a consequence of fiber bridging the toughness increased approximately linearly with crack length. The slopes of the $R$ curves were fit to numerical simulations to determine a cohesive fiber bridging stress.

\section{Materials and Methods}

\section{Medium Density Fiberboard (MDF) Fracture Specimens}

The MDF panels were provided by Flakeboard ${ }^{\circledR}$ (Springfield, OR) as $4 \mathrm{ft} X 8 \mathrm{ft}$ panels at two densities, 38 and $46 \mathrm{lbs} / \mathrm{ft}^{3}\left(609\right.$ and $\left.737 \mathrm{~kg} / \mathrm{m}^{3}\right)$ and in two thicknesses, 0.5 and 0.75 in (12.7 and $19.05 \mathrm{~mm}$ ). Prior to testing, all panels were conditioned at $20^{\circ} \mathrm{C}$ and $65 \%$ relative humidity until equilibrium.

Figure 1a shows an extended version of the ASTM E399 (2006) compact tension specimen (CT). Extra length was used to allow more room for crack propagation. All specimens used $W=$ 3 in and $\Delta=1.25 \mathrm{in}$. Although in-plane properties of MDF are nominally isotropic, manufacturing processes may cause differences between the long (Longitudinal) and short (Transverse) axis of the panel. Between in-plane and thickness directions, the properties differ and the fracture properties are expected to differ too. To measure anisotropy effects on toughness, specimens were cut for four different propagation directions: TL, LT, ZL, and ZT (see Fig. 1b). Here $\mathrm{L}$ is the long or $8 \mathrm{ft}$ direction of the initial panel, $\mathrm{T}$ is the short or $4 \mathrm{ft}$ direction, and $\mathrm{Z}$ is the thickness direction. Specimens for TL and LT cracks (denoted as in-plane cracks) were cut from the panel as illustrated in Fig 1b. For ZL and ZT cracks (denoted as Z cracks), square slices of the panel (with square sides equal to the panel thickness) were cut in the $\mathrm{L}$ and $\mathrm{T}$ directions for length of $1.25 W+\Delta=5$ in (see Fig 1b). These slices were glued between 
in-plane pieces of the same panel to create an extended CT geometry such that ZL or ZT cracks propagated down the center of the slice. The remaining orthogonal cracks LZ and TZ were not tested because the $\mathrm{Z}$ direction is too short for crack propagation experiments. The pin-loaded specimens were loaded in a $10 \mathrm{kN}$ Sintech testing frame. Displacements were measured using Eplison $^{\circledR}$ clip gauge-style extensometer attached at the pin-loading line.

\section{Crack Length Measurement}

Analysis of crack propagation experiments requires accurate measurement of crack length during the experiment. In many materials crack length can easily be detected on the surface, but that approach does not work for MDF. To measure MDF crack lengths, we used Digital Image Correlation (DIC, Correlated Solutions Inc., West Columbia, SC) or digital image processing (Samarasinghe, 1999). The theory and setup procedure of DIC systems, also called electronic speckle photography, is described well by Sutton, 1983.

Using DIC, strain fields ahead of the crack tip were monitored throughout the loading. For example, Fig. 2 shows the axial strain, or the strain in the direction of loading, along the crackline for a series increasing crack lengths (from left to right). The strain profiles were high near the crack tip and decreased as a function of distance away from the crack tip. Identifying an absolute crack tip was impossible, but between two images a $\Delta a$, or increment in crack growth, could be accurately measured from the shift in the strain profile. Since the energy methods described below only require $\Delta a$, and not an absolute crack length, the DIC results were sufficient for fracture toughness experiments. 


\section{Fracture Toughness by Energy Analysis}

In an unloading curve following in increment of elastic fracture returns to the original origin (Fig. 3, left), the fracture energy is the area within the triangular area $\mathrm{ABC}$ (Fig. 3, right) between loading and unloading curves. The fracture toughness is the energy per unit fracture area. This fact applies regardless of the presence of fiber bridging. The toughness from a discrete observation of crack growth, $\Delta a=a_{j}-a_{i}$, can be calculated various ways. Two convenient methods are (Hashemi, et al., 1990):

$$
G_{c}=\frac{P_{i}\left(u_{j}-u_{0}\right)-P_{j}\left(u_{i}-u_{0}\right)}{2 B \Delta a} \quad \text { and } \quad G_{c}=\frac{P_{i} P_{j}\left(C_{j}-C_{i}\right)}{2 B \Delta a}
$$

where $P_{i}$ is the load when the crack of length $a_{i}$ starts to propagate at displacement $u_{i}$ and $P_{j}$ is the load when crack propagation stops at length $a_{j}$ and displacement $u_{j} . C_{i}$ and $C_{j}$ are the specimen compliances before and after crack propagation; $u_{0}$ is the displacement at the start of the test. These equations can calculate toughness as a function of crack propagation from a collection of discrete results for $P_{i}, a_{i}, u_{i}$ and/or $C_{i}$ during an experiment. Hashemi, et al. (1990), for example, used this approach for analysis of composite delamination toughness.

For a linear-elastic material with negligible plasticity, unloading curves after crack propagation should return to the origin. In these MDF experiments, however, they returned to a positive displacement (see $u_{R}$ in Fig 3, right). There are three possible reasons for a positive displacement offset: residual stresses, plasticity, or crack-plane interference (Atkins and Mai, 1988). A residual stress effect could be ruled out by specimen analysis (Nairn, 1997, 1999). A plasticity effect would invalidate elastic fracture analysis, but a crack-plane interference effect may not. Crack-plane interference means the bridging material left in the wake of the crack cannot be unloaded back to the original specimen configuration. Instead, the bridging material is 
crushed causing the unloading compliance to be lower, resulting in a residual displacement. Thus, a residual displacement is possible even when the fracture process is entirely elastic.

A test proposed by Atkins and Mai (1988) was used to distinguish between plasticity and crack-plane interference. First, a specimen was loaded at a rate of $0.5 \mathrm{~mm} / \mathrm{min}$ until the crack had propagated through roughly half of the previously un-cracked ligament length and the loading was paused. Next, the fiber-bridging zone around the crack tip was removed. The crack line was drilled out using a $1 / 8^{\text {th }}$ inch bit. The remaining bridging material was cut out with a razor blade and saw. Finally, the specimen was unloaded. If a specimen with the zone removed returns to the origin, then the residual displacements can be attributed to crack-plane interference. In MDF specimens, removing the process zone reduced the residual displacement by about $90 \%$ (Matsumoto and Nairn, 2007). By necessity, comparisons between not removing and removing the zone had to be done on different specimens. It was therefore challenging to unambiguously determine the amount of crack-plane interference. Our results, however, strongly suggested that crack-plane interference is present in MDF and is the major cause of residual displacements.

When crack-plane interference is present, the second approach in Eq. (1) cannot be used. The measured $C_{i}$ would differ from the true unloading $C_{i}$ due to the crack-plane interference. Furthermore crushing of material in the process zone during unloading might influence subsequent crack propagation. The first approach in Eq. (1) can still be used provided the specimen is never unloaded. Subsequent tests therefore monotonically increased the load, recorded load $v s$. displacement, and monitored the crack length using DIC. Each adjacent pair of crack length results was substituted into Eq. (1) to determine $G_{c}$ as a function of crack length ( $R$ curve). We termed this approach the discrete analysis for toughness. 


\section{Revised R-Curve Analysis}

Discrete $R$-curve experiments have been used before, but the method is prone to scatter (Hashemi et al., 1990). The numerical difficulty is that it relies on subtraction of similar numbers and precise determination of $\Delta a$. A revised analysis method was recently developed that reduces the scatter by eliminating the need to divide by $\Delta a$ (Nairn, 2008). First, load and crack length $v s$. displacement data sets are obtained during monotonic loading. Next, the cumulative energy released, $U(d)$, per unit specimen thickness, $B$, is found by integrating force-displacement data, $F(d)$, up to some displacement $d$ and subtracting the area under an assumed elastic return to the origin (see Fig. 4A):

$$
U(d)=\frac{1}{B}\left(\int_{0}^{d} F(x) d x-\frac{1}{2} F(d) d\right)
$$

Next, the crack length data are fit (and smoothed if necessary) to get crack length as a function of displacement, $a(d)$. By treating displacement as a parametric variable, the results for $U(d)$ and $a(d)$ are recast as cumulative energy released as a function of crack length, $U(a)$. This integral transformation always gives smooth curves from actual fracture experiments. The $R$ curve is found by numerically differentiating $U(a)$ :

$$
R=\frac{d U(a)}{d a}
$$

The numerical differentiation step usually benefits by smoothing. Here we used simple running average of the tangent to the curve. The running average window was typically $1 / 3$ to $1 / 4$ of the range of the crack length data. The process is illustrated graphically in Fig. 4. 


\section{Material Point Method Modeling}

The material point method (MPM) was used to model experimental results. MPM was developed as a numerical method for solving problems in dynamic solid mechanics (Sulsky et al., 1994, 1996). In MPM, a solid body is discretized into a collection of points much like a computer image is represented by pixels. As the dynamic analysis proceeds, the solution is tracked on the material points by updating all required properties such as position, velocity, acceleration, stress state, etc..

The meshless nature of MPM recommends it for analysis of explicit cracks including crack propagation with process zones. Although early MPM did not allow cracks, it was extended to CRAMP, which signifies CRAcks in the Material Point method (Nairn, 2003; Guo and Nairn, 2004, 2006) and models explicit cracks. The crack plane is defined by a linked series of massless particles that translate through the grid along with the material points. Crack propagation is modeled by adding a new crack particle at the crack tip. The propagation process is not constrained by any mesh and therefore can follow an arbitrary path. Recently the CRAMP algorithm was extended further to all traction laws on the crack particles (Nairn, 2008). This new approach allows simulations of cracks with cohesive zones or cracks with a combination of crack tip processes and a process zone described by a traction law. Unlike most finite element analysis with cohesive zones, the traction law zones in MPM do not have to be inserted prior to the analysis. They develop naturally as the crack propagation process proceeds. This key feature was used to simulate $R$ curves as the fiber-bridging process zone developed.

\section{Results}

Figure 5 shows $R$ curve results for LT fracture in $0.5 \mathrm{in}, 38 \mathrm{lbs} / \mathrm{ft}^{3}$ panels analyzed two different ways. The symbols used a discrete analysis (Eq. (1)) for each pair of DIC images 
analyzed for crack growth. The smooth curve used the revised $R$-curve analysis. The revised method is the slope of the cumulative energy per unit thickness transformation on raw data by Eq. (2); that cumulative energy is plotted in Fig. 6 (dashed 38 LT curve). Both results are the average of results from three specimens. The two analysis methods agree, but the revised method has less scatter. Both methods indicate a steady rise in toughness until the crack approaches the end of the specimen, which is followed by a rapid rise. The slow rise is clearer in the revised analysis method. Our interpretation is that the $R$ curve for MDF increases roughly linearly with crack length. The rapid rise at the end was either due to edge effects or to numerical artifacts caused by $\Delta a$ approaching zero near the end of the test. A small $\Delta a$ makes accurate measurement of $R$ difficult.

Figures 6 and 7 have results for all LT and TL fracture results. Figure 6 has the cumulative energy transformation of the raw data. Figure 7 has derivatives of the raw energy data to give the material's $R$ curve as a function of crack growth. For all specimens, the toughness starts at a high value and then increases linearly with crack length. The toughness of the higher density panels $\left(46 \mathrm{lb} / \mathrm{ft}^{3}\right)$ is more than double the toughness of the lower density panels $\left(38 \mathrm{lbs} / \mathrm{ft}^{3}\right)$. The solid and dashed lines are results for thicker ( 0.75 in) and thinner $(0.5$ in) panels, respectively. For the higher density panels, the initial toughness was independent of density, but the slope of the $R$ curve was higher for the thinner panels. For the lower density panels, the toughness and slope were independent of thickness, although the thinner specimen had a slightly higher slope.

There was little difference between TL and LT fracture in the $48 \mathrm{lbs} / \mathrm{ft}^{3}$ panels. Those results were therefore averaged to get in-plane $R$-curve results as summarized in Table 1 . There are no results for TL fracture in the $38 \mathrm{lb} / \mathrm{ft}^{3}$ panels. Cracks in that orientation turned and therefore could not be analyzed. The results in table 1 are the average of three LT specimens. 
Figures 8 and 9 give $R$ curves for $Z$ cracks. Like the in-plane cracks, the toughness had an initial value and then increased linearly with crack growth. Both the initial value and the slopes are 50-100 times lower for the $Z$ cracks than for the in-plane cracks. There was little influence of thickness for the $38 \mathrm{lbs} / \mathrm{ft}^{3}$ panels. For the $46 \mathrm{lbs} / \mathrm{ft}^{3}$ panels, the thinner specimens had higher toughness and slope. No systematic effect of ZL $v s$. ZT was detected. The results are averaged and summarized in Table 1.

\section{Discussion}

\section{Prior Toughness Results}

Very few experiments for the fracture toughness of MDF have been done. Niemz et al. $(1997,1999)$ used ASTM methods (2006) to measure stress intensity factor for the initiation of crack growth. They found MDF (of $710 \mathrm{~kg} / \mathrm{m}^{3}$ density) to have a $K_{I C}=1.81 \mathrm{MPa} \sqrt{\mathrm{m}}$. Assuming the MDF in-plane modulus is about $3000 \mathrm{MPa}$ and it's Poisson's ratio is 0.33 (Ganev et al., 2005), this stress intensity is equivalent to $G_{c}=970 \mathrm{~J} / \mathrm{m}^{2}$. This result is lower than our panels of similar density. Furthermore, because the ASTM method assumes self-similar crack growth and stress-free surfaces, while MDF cracks have fiber bridging, the ASTM approach does not give a valid toughness (Matsumoto et. al, 2007).

Morris et al. (1999) used the Nordtest (Larsen and Gustafsson, 1990, 1993) method and found MDF (of $800 \mathrm{~kg} / \mathrm{m}^{3}$ ) to have $\mathrm{a} \mathrm{G}_{\mathrm{IC}}=5918 \mathrm{~J} / \mathrm{m}^{2}$. The Nordtest integrates the forcedisplacement curve to find total energy to propagate a crack along the entire specimen. It should be similar to an average of an $R$ curve over the entire specimen length; their results are similar to our higher-density $R$ curves. Two problems with total fracture energy are that it does not give information about rising $R$ curves and it may be disproportionately affected by edge effects (e.g., the high $R$ values near the end of the crack growth in Fig. 7). We claim direct measurement of 
energy released during crack growth, as done here, is preferred over total integrated energy methods.

Ehart, et. al (1996) did crack propagation in particle board — a material analogous to MDF but composed of wood particles rather than find wood fibers. They measured the energy between load-displacement curves of different specimens that they perceived to have the same amount of fiber bridging. An effective crack length for each specimen (with fiber bridging) was determined through finite element analysis. The use of an effective crack length limits such results to being an effective toughness. The DIC methods used here eliminated the need to rely on effective crack lengths. The DIC approach should work or particle board as well as for MDF.

\section{Fiber Bridging Analysis}

The usual starting point for analysis of fracture with bridging is to equate $R$ to the sum of crack-tip processes and fiber bridging processes (Rice 1968, Bao and Suo, 1990):

$$
R_{s s}=G_{t i p, c}+\int_{0}^{\delta_{c}} \sigma(\delta) d \delta
$$

where $G_{t i p, c}$ is the toughness associated with crack-tip processes and the integral is the energy associated with fiber bridging. The fiber bridging energy is the area under the fiber bridging traction $(\sigma(\delta))$ - crack opening displacement (COD or $\delta$ ) law, where $\delta_{c}$ is the COD where the bridged fibers fail (see Fig. 10). But, Eq. (4) is only valid for steady-state crack propagation after the bridging zone is fully developed and the $R$ curve has reached a constant toughness. Steadystate crack propagation is also self-similar crack propagation because the process zone size remains constant as the crack propagates. Thus, like many fracture standards, the usual starting point for fiber-bridging analysis implicitly assumes self-similar crack growth. 
All crack propagation in MDF, however, was within the rising portion of the $\mathrm{R}$ curve and is thus non-self-similar crack growth. Modeling the $R$ curve in this regime requires additional knowledge (or assumptions) about the fiber-bridging process. One recent model is to assume the bridging law describes elastic processes, but can soften as the bridging fibers fail (Nairn, 2008). Under this assumption, non-steady-state $R$ curve can be modeled with (Nairn, 2008)

$$
R_{n s s}=G_{t i p, c}+\int_{0}^{\delta_{n}} \sigma(\delta) d \delta-\frac{1}{2} \sigma\left(\delta_{n}\right) \delta_{n}
$$

This model is illustrated in Fig. 10A. The shaded area is the sum of the last two terms. The integral is the area up to the current crack opening displacement, $\delta_{n}$. The last term subtracts off the elastic energy stored in the bridging zone that has not been released to fracture and thus is not part of the observed $R$. Daudeville (1999) similarly treated fiber bridging in solid wood as an process zone with softening due to development of damage. He modeled force-displacement curves during crack propagation, but did not consider analysis of rising $\mathrm{R}$ curves and attributed the entire toughness to bridging (i.e., assumed $G_{t i p, c}=0$ ).

Examination of several traction laws (Nairn, 2008) shows that a linear increase in $R$ is characteristic of a linear softening law (see Fig. 10B) where the bridging traction rises rapidly to a peak stress or cohesive stress $\left(\sigma_{c}\right)$ and then decreases linearly to zero at the critical COD $\left(\delta_{c}\right)$. The area under the bridging law is the bridging toughness, $G_{B}$. Our hypothesis was that MDF fiber bridging has such a linear softening law. The question remains — what traction law properties can be determined from the experimental results? The fiber bridging toughness, $G_{B}$, can be determined from the difference between initial $R$ and the steady-state $R$. Since these MDF experiments never reached steady state, $G_{B}$ could not be determined. Analysis of $R$ curves with 
linear softening shows the slope is related to the peak cohesive stress, $\sigma_{c}$. The determination of fiber-bridging cohesive stress in MDF required numerical modeling.

Crack propagation with fiber bridging in MDF was modeling using the material point method (MPM). The details are in Nairn (2008). In brief:

1. An explicit crack was introduced into the MPM model.

2. As the calculations proceeded, the crack-tip energy release rate, $G_{t i p}$, was calculated by $J$ integral methods that account for bridging effects (Nairn, 2008). When $G_{t i p}>G_{t i p, c}$, the physical crack tip propagated by MPM methods for explicit crack propagation (Nairn, 2008). The newly created crack surface area was assigned to the selected, linear-softening traction law with the COD initialized to zero.

3. At the time of crack propagation, the total fracture energy released was calculated using Eq. (5).

4. On each time step, the COD along the crack surface was calculated and bridging fibers failed whenever $\mathrm{COD}>\delta_{c}$.

5. The calculations continued until the crack length reached the end of the specimen. A plot of $R$ (from step 3 ) was compared to experimental results.

Figure 11 compares simulations to experimental results (for LT fracture in $0.75 \mathrm{in}, 38 \mathrm{lb} / \mathrm{ft}^{3}$ panels) for various values of cohesive stress, $\sigma_{c}$, and bridging toughness, $G_{B}$. The open symbols are for $\sigma_{c}=0.25,0.45$, and $1 \mathrm{MPa}$ with $G_{B}=500 \mathrm{~J} / \mathrm{m}^{2}$; all simulations used $G_{t i p, c}=2210 \mathrm{~J} / \mathrm{m}^{2}$. All simulated $R$ curves are linear in crack length and the slope increased with $\sigma_{c}$. A value of $\sigma_{c}=$ $0.45 \mathrm{MPa}$ agreed with the experimental results. For $\sigma_{c}=1 \mathrm{MPa}$, the simulations reached steady state at a crack length of about $50 \mathrm{~mm}$. The steady state toughness is greater than the initial toughness by the input $G_{B}$. The simulations for $\sigma_{c}=0.25$ and $0.45 \mathrm{MPa}$ did not reach steady state 
prior to the end of the sample. The filled symbols are for $\sigma_{c}=0.45 \mathrm{MPa}$ with $G_{B}=200 \mathrm{~J} / \mathrm{m}^{2}$. Because of the lower $G_{B}$, this simulation reached steady state at a crack length of about $50 \mathrm{~mm}$. The initial slope, however, was identical to the other $\sigma_{c}=0.45 \mathrm{MPa}$ simulation, which confirms that the slope is a function only of $\sigma_{c}$. Therefore $\sigma_{c}$ could be determined from the slope of experimental results without knowledge of $G_{B}$.

The results for $\sigma_{c}$ determined by numerical simulation are given in Table 1 . The cohesive stress increased with density and is usually higher for the thinner panels. The cohesive stress for in-plane crack propagation is about and order of magnitude higher than for Z-crack propagation. Although $G_{B}$ could not be determined, it can be bounded. From the increment in energy prior to edge effects $G_{B}$ is greater than $1000 \mathrm{~J} / \mathrm{m}^{2}$ and $500 \mathrm{~J} / \mathrm{m}^{2}$ for thin and thick, $38 \mathrm{lb} / \mathrm{ft}^{3}$ panels, and greater than $3000 \mathrm{~J} / \mathrm{m}^{2}$ and $1000 \mathrm{~J} / \mathrm{m}^{2}$ for thin and thick $46 \mathrm{lb} / \mathrm{ft}^{3}$ panels. For $\mathrm{Z}$ cracks $G_{B}$ is greater than $11 \mathrm{~J} / \mathrm{m}^{2}$ and $15 \mathrm{~J} / \mathrm{m}^{2}$ for thin and thick, $38 \mathrm{lb} / \mathrm{ft}^{3}$ panels, and greater than $40 \mathrm{~J} / \mathrm{m}^{2}$ and $15 \mathrm{~J} / \mathrm{m}^{2}$ for thin and thick $46 \mathrm{lb} / \mathrm{ft}^{3}$ panels. The cohesive stress, bridging toughness, and critical bridging COD are related by

$$
G_{B}=\frac{1}{2} \sigma_{c} \delta_{c}
$$

Although $\delta_{c}$ could be determined, it can also be bounded from the bounds on $G_{B}$. The lower bound for $\delta_{c}$ was found to be independent of both thickness and density. For the in-plane cracks $\delta_{c} \geq 2.57 \pm 0.22 \mathrm{~mm}$. For $Z$ cracks $\delta_{c} \geq 0.50 \pm 0.13 \mathrm{~mm}$. The in-plane $\delta_{c}$ is comparable to the expected fiber length in MDF made from softwood of 3 to $4 \mathrm{~mm}$ (Bower et al., 2003). The $\delta_{c}$ for $\mathrm{Z}$ cracks is much lower, probably because MDF fibers tend to lie flat in the plane due to mat compaction used during manufacturing (Bower et al., 2003). 
Most fracture models in finite element analysis use either fracture mechanics criteria or cohesive zones model (CZM) (Needleman, 1987). A conventional fracture mechanics model cannot handle process zones. In conventional CZM methods, the cohesive elements have to be inserted prior to the analysis. Since cohesive elements need to cross the entire specimen, there is no crack tip that can be monitored for crack length to compare to experiments. The MPM modeling here is a generalization of fracture modeling to use both fracture mechanics and a process zone. Fracture mechanics methods were used to model crack-tip processes (Nairn, 2008). Traction-law methods were used to model the bridging zone, but they were not inserted prior to the analysis. They were inserted only as the crack propagated.

\section{Implications for General Composite Fracture}

If a fiber-bridging zone (or any kind of process zone) is not small compared to specimen dimensions, the fracture process will be influenced by that zone. At the beginning of crack propagation, the process zone develops and the toughness of the material will change. This effect leads to a rising $R$ curve. The shape of the $R$ curve depends on the mechanics of the process zone (Nairn, 2008). During a rising $R$ curve, standard methods that assume self-similar crack propagation cannot be used. If the process zone has elastic processes, however, elastic fracture mechanics is still valid, but the toughness has to be measured directly.

Fracture mechanics standards like ASTM E399 (2006) start with a machined notch with no

process zone. The toughness is determined from the load for initiation of the crack and no information about crack propagation is recorded. A common misconception is that the subsequent process zone does not influence this initial crack growth and therefore the standard gives a valid initiation result. The energy released for crack initiation, $G_{c}$, is given by 


$$
G_{c}=\frac{P^{2}}{2 B} \frac{d C(a)}{d a}
$$

where $P$ is the load at initiation, $B$ is thickness, and $C(a)$ is the specimen compliance as a function of crack length, $a$. Whenever crack propagation results in a process zone, $d C(a) / d a$ will be influenced by that zone, it will even be influenced for initiation or in the limit $a \rightarrow a_{0}$. Since the calibration functions in ASTM E399 (2006) use numerical methods that effectively calculate $d C(a) / d a$ under the assumption of no process zone (Gross et al., 1964), those functions will give an invalid result when a process zone occurs. Another danger of initiation experiments in standard methods is that the subsequent crack propagation is ignored. There is no way to tell from an initiation load whether or not a process zone has affected the results. In other words, fracture toughness of composites should never be measured by conventional methods unless those methods are supplemented with crack propagation experiments and those propagation experiments show there is no process zone or only a negligible process zone (Matsumoto and Nairn, 2007).

When composite fracture requires direct measurement of toughness, several experimental difficulties may arise, and many arose in experiments on MDF. For example, a common effect of a fiber-bridging zone is crack-plane interference. When interference is present, unloading steps cannot be used. The test has to be conducted monotonically with continuous monitoring of crack length. Another problem in direct measurement of energy is avoiding scatter caused by taking differences between discrete data points. Indeed much work on composite delamination fracture is aimed at developing refined beam theories with the primary goal being to avoid analysis by Eq. (1) (Hashemi et al., 1990). The revised energy method used here shows promise for ameliorating problems with direct energy measurements. It may be possible to refine the method 
by optimization of the transformation process (e.g., by constraining the analysis to produce a monotonically increasing and smooth $R$ curve).

Finally, one approach to materials with process zones is to ignore the rising portion of the $R$ curve and characterize the toughness from the constant value during steady-state crack propagation. This approach has two problems. First, many composite materials may never reach steady state crack propagation within the chosen fracture specimen. This situation applies to MDF specimens used here. The characterization of such materials requires methods that can be used during non steady-state propagation or possibly much longer specimens such that steady state conditions can be observed. Second, the shape of the rising R curve gives material property information about the mechanics of the process zone. Techniques, such as the simulations used here, can extract material property information from the rising $\mathrm{R}$ curve.

\section{Acknowledgements}

We thank David Kruse, from Flakeboard, for providing all the MDF panels and Milo

Clauson, Oregon State University, for much help on mechanical testing and DIC methods. Noah Matsumoto was support by a USDA Wood Utilization Special Research Grant.

\section{References}

Annual book of ASTM Standards (2006), "Standard Test Method for Plane-Strain Fracture Toughness of Metallic Materials," ASTM Designation: E399-05a.

Atkins, A.G., Mai, Y-W. (1988). "Elastic and Plastic Fracture Mechanics: metals polymers, composites, biological materials." John Wiley and Sons, Ellis Horwood Limited, Market Cross House, Cooper Street, Chichester, West Sussex, PO19 1EB, England, 108-113.

Bao G., Suo Z. (1992) “Remarks on Crack-Bridging Concepts,” Appl. Mech. Rev., 45 (6), 355366.

J. Bodig and B. A. Jayne (1982). Mechanics of Wood and Wood Composites. Van NostranReinhold Co, Inc., New York. 
Bower, J.L., Shmulsky, R. and Haygreen, JG. (2003). "Forest Products and Wood Science-An Introduction." $4^{\text {th }}$ Edition. Ames, IA: Iowa State Press.

Daudeville, L. (1999). "Fracture in Spruce: Experiment and Numerical Analysis by Linear and Non Linear Fracture Mechanics.” Holz als Roh - und Werkstoff, 57, 425-432.

Ehart, R.J., Stanzl-Tschegg, S.E., Tschegg, E.K. (1996). “Characterization of Crack Propagation in Particleboard.” Wood Science and Technology 30, 307-321.

Ganev, S., Gendron G., Cloutier, A., Beauregard, R. (2005). "Mechanical Properties of MDF as Function of Density and Moisture Content." Wood and Fiber Science, Vol. 37, 2, 314226.

Gross, B., Srawley, J.E., Brown, W.F. (1964). "Stress Intensity Factors for a Single-EdgeNotched Tension Specimen by Boundry Collocation of a Stress Function.” NASA Lewis Research Center.

Guo Y., Nairn J. A. (2004). “Calculation of J-Integral and Stress Intensity Factors Using the Material Point Method." Computer Modeling in Engineering \& Sciences, 6, 295-308.

Guo Y., Nairn J. A. (2006) . "Three-Dimensional Dynamic Fracture Analysis in the Material Point Method." Computer Modeling in Engineering \& Sciences, 16, 141-156.

Hashemi, S., Kinloch A.J., William, J.G. (1990). "The Analysis of Interlaminar Fracture in Uniaxial Fibre Reinforced Composites.” Proc. R. Soc. London, A347, 173-199.

Larsen, H.J., Gustafsson, P.J. (1990). "The Fracture Energy of Wood in Tension Perpendicular to the Grain." 23th CIB-W18 A Meeting. Lisbon Portugal, Paper 23-19-2.

Larsen, H.J., Gustafsson, P.J. (1993). "Determination of Fracture Energy of Wood for Tension Perpendicular to the Grain." Draft NORDTEST Method, Lund Institute of Technology, Lund Sweden.

Matsumoto, N., Nairn, J. (2007). "Fracture Toughness of MDF and other Materials with Fiber Bridging." Proc. of $22^{\text {nd }}$ Ann. Tech. Conf. of the American Society of Composites, Sept 17-19, Seattle, WA.

Morris, V.L., Hunt, D.G, Adams, J.M. (1999). "The Effects of Experimental Parameters on the Fracture Energy of Wood-based Panels" Journal of the Institute of Wood Science 85, 32-

J. A. Nairn (1997), "Fracture Mechanics of Composites with Residual Thermal Stresses," J. Appl. Mech., 64, 804-810.

J. A. Nairn (1999), "Energy Release Rate Analysis of Adhesive and Laminate Double Cantilever Beam Specimens Emphasizing the Effect of Residual Stresses," Int. J. Adhesion \& Adhesives, 20, 59-70. 
Nairn J. A. (2003) "Material Point Method Calculations with Explicit Cracks. Computer Modeling in Engineering \& Sciences, 4, 649-664.

J. A. Nairn (2008). "Analytical and Numerical Modeling True R Curves for Cracks with Process Zones Using J Integral Methods", Int. J. Fracture, submitted.

Needleman A. (1987). "A Continuum Model for Void Nucleation by Inclusion Debonding." J. Appl. Mech., 54, 525-531

Niemz, P., Diener, M. and Pöhler, E. (1997). "Untersuchungen zur Ermittlung der Bruchzähigkeit an MDF-Platten," Holz als Roh- und Werkstoff, 55 (5), 327-330.

Niemz, P., Diener, M. and Pöhler, E. (1999). "Vergleichende Untersuchungen xur Ermittlung der Bruchzahigkeit an Holzwerkstoffen," Holz als Roh- und Werkstoff, 57, 222-224.

Rice, J. R. (1968). “A Path Independent Integral and the Approximate Analysis of Strain Concentration by Notches and Cracks," J. Applied Mech., June, 379-386

Samarasinghe, S., Kulasiri, G.D. (2000). "Displacement fields of wood in tension based on image processing: Part 1." Silva Fennica, 34(3), 251-259.

Sulsky D., Chen Z., Schreyer H. L. (1994). “A Particle Method for History-Dependent Materials.” Comput. Methods. Appl. Mech. Engrg., 118, 179-186.

Sulsky D., Zhou S. J., Schreyer H. L. (1995). “Application of a Particle-in-Cell Method to Solid Mechanics." Comput. Phys. Commun., 87, 236-252.

Sutton, M.A., Wolters, W.J., Peters, W.H., Rawson, WF., and McNeil, S.R. (1983). "Determination of displacement using an improved digital image correlation method." Image and Vision Computing, 1(3), 133-139. 


\section{Tables}

Table 1: The initiation toughness $\left(G_{c}\right)$ and slope of the rising $R$ curve for two densities and two thicknesses of MDF panels. The "in plane" cracks average the LT and TL results. The "Z cracks" average the ZL and ZT results.

\begin{tabular}{|l|c|c|c|c|c|c|}
\cline { 2 - 7 } \multicolumn{1}{c|}{} & \multicolumn{3}{c|}{ Thin $(0.5$ in $)$} & \multicolumn{3}{c|}{ Thick $(0.75$ in) } \\
\hline Panel/Crack Type & $\begin{array}{c}G_{c} \\
\left(\mathrm{~J} / \mathrm{m}^{2}\right)\end{array}$ & $\begin{array}{c}\text { Slope } \\
\left(\mathrm{J} / \mathrm{m}^{3}\right)\end{array}$ & $\begin{array}{c}\sigma_{c} \\
(\mathrm{MPa})\end{array}$ & $\begin{array}{c}G_{c} \\
\left(\mathrm{~J} / \mathrm{m}^{2}\right)\end{array}$ & $\begin{array}{c}\text { Slope } \\
\left(\mathrm{J} / \mathrm{m}^{3}\right)\end{array}$ & $\begin{array}{c}\sigma_{c} \\
(\mathrm{MPa})\end{array}$ \\
\hline $38 \mathrm{lbs} / \mathrm{ft}^{3}$, in plane & 2062 & 21700 & 0.79 & 2233 & 10500 & 0.43 \\
\hline $38 \mathrm{lbs} / \mathrm{ft}^{3}, \mathrm{Z}$ cracks & 54.0 & 222 & 0.038 & 48.2 & 296 & 0.056 \\
\hline $46 \mathrm{bs} / \mathrm{ft}^{3}$, in plane & 4153 & 59600 & 2.55 & 4452 & 18400 & 0.66 \\
\hline $46 \mathrm{bs} / \mathrm{ft}^{3}, \mathrm{Z}$ cracks & 75.3 & 814 & 0.14 & 48.4 & 303 & 0.10 \\
\hline
\end{tabular}

\section{Figure Captions}

Figure 1: a. Extended compact tension specimen where $a=1.2 "(30.48 \mathrm{~mm}), W=3 "(76.2 \mathrm{~mm})$, and $\Delta=1.25 "(31.75 \mathrm{~mm})$. A $\Delta$ of zero is the ASTM CT specimen, but here a non-zero $\Delta$ was used to allow more crack propagation. b. Specimen orientation in a panel (not to scale).

Figure 2: Profiles for axial strain as a function of position along the crack-line obtained from DIC. Curve 1 is prior to crack growth. Curves 2 to 7 are profiles after subsequent increments in crack growth. The shift between curves was a measurement of the amount of crack growth between those two points in the test.

Figure 3: Left: load displacement curve for elastic fracture where the test is periodically stopped and unloaded. Right: A single loading and unloading envelop. Elastic fracture follows path $\mathrm{ABC}$. Fracture with residual displacements follows path ABCD.

Figure 4: Graphical illustration of the revised $R$-curve method. The left shows integral transformation of force and crack length data as a function of displacement to cumulative energy as a function of crack length (B). C shows the $R$ curve as found from the slope of the energy area.

Figure 5: Analysis of the $R$ curve for $0.5 \mathrm{in}, 38 \mathrm{lb} / \mathrm{ft}^{3}$, LT fracture by two different methods. The symbols used the discrete method. The smooth line used the revised $R$-curve analysis method. 
Figure 6: The cumulative energy released per unit thickness, $U(a)$, for all in-plane fracture specimens. The dashed lines are for 0.5 in thick panels; the solid lines are for 0.75 in thick panels.

Figure 7: $R$ curves for all in-plane fracture experiments. The dashed lines are for 0.5 in thick panels; the solid lines are for 0.75 in thick panels.

Figure 8: $R$ curves for all $Z$ cracks in the $38 \mathrm{lb} / \mathrm{ft}^{3}$ panels. The dashed lines are for 0.5 in thick panels; the solid lines are for 0.75 in thick panels.

Figure 9: $R$ curves for all $Z$ cracks in the $46 \mathrm{lb} / \mathrm{ft}^{3}$ panels. The dashed lines are for 0.5 in thick panels; the solid lines are for 0.75 in thick panels.

Figure 10: Fiber-bridging traction laws used to model the fiber bridging process zone. The shaded area in A illustrates the concept of energy released from the process zone prior to steady state crack propagation. B shows the linear softening law used to model fiber bridging in MDF.

Figure 11: Comparison of simulation results (symbols) to experimental results (sold line) for LT crack growth in $0.75 \mathrm{in}, 38 \mathrm{lb} / \mathrm{ft}^{3}$ panels. The open symbols are for different values of $\sigma_{c}$ and $G_{B} \geq 500$. The solid symbols used a lower $G_{B}$. 

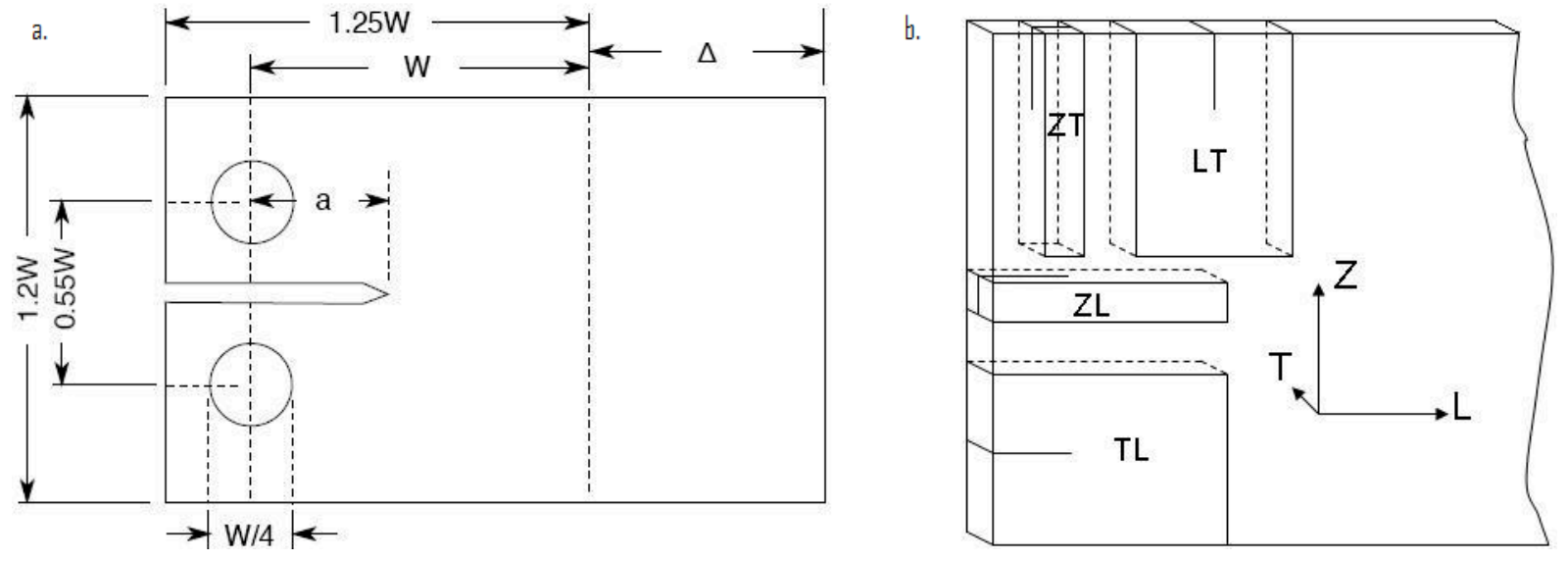

Figure 1: a. Extended compact tension specimen where $a=1.2 "(30.48 \mathrm{~mm}), W=3 "(76.2 \mathrm{~mm})$, and $\Delta=1.25 "(31.75 \mathrm{~mm})$. A $\Delta$ of zero is the ASTM CT specimen, but here a non-zero $\Delta$ was used to allow more crack propagation. b. Specimen orientation in a panel (not to scale). 


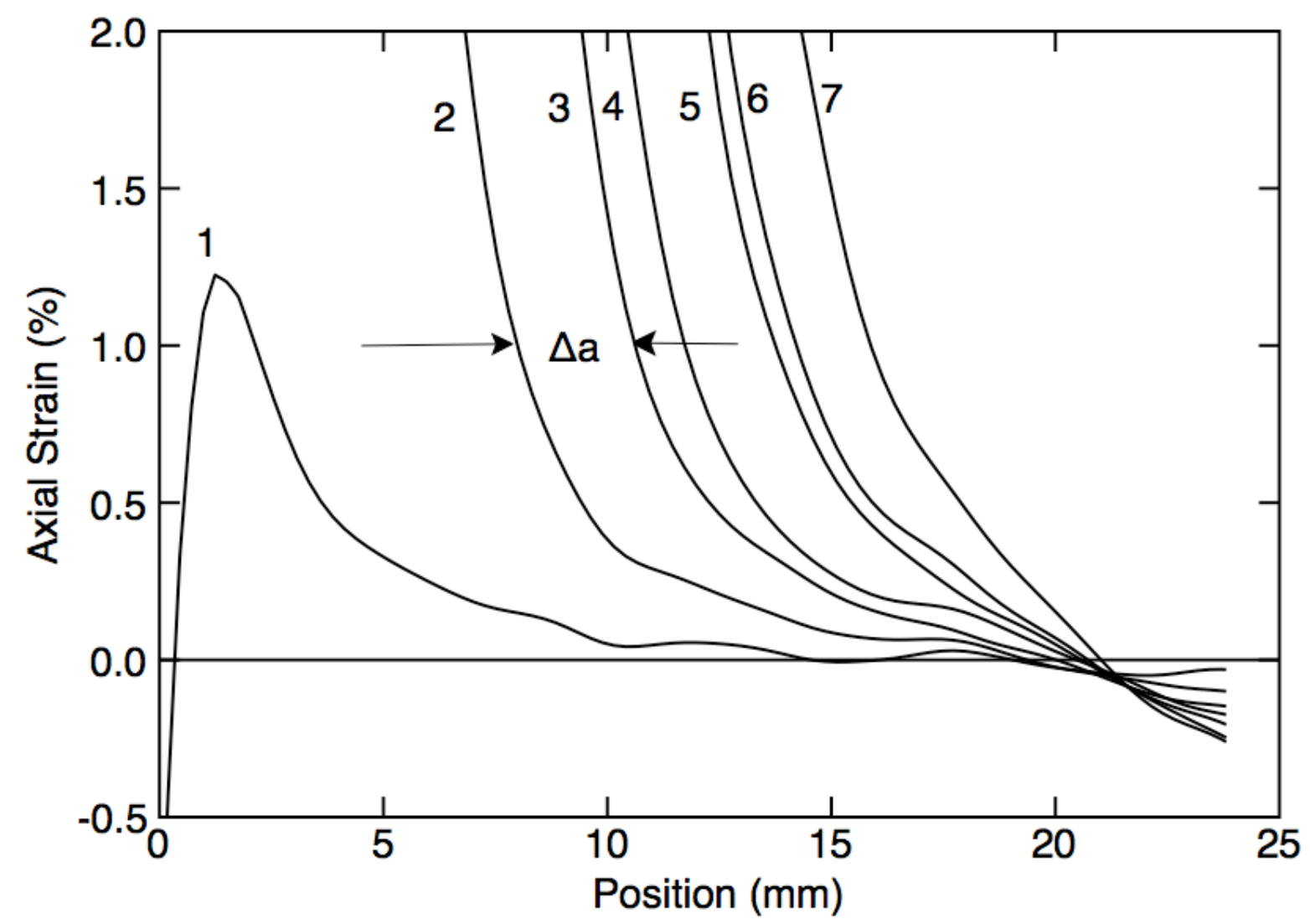

Figure 2: Profiles for axial strain as a function of position along the crack-line obtained from DIC. Curve 1 is prior to crack growth. Curves 2 to 7 are profiles after subsequent increments in crack growth. The shift between curves was a measurement of the amount of crack growth between those two points in the test. 

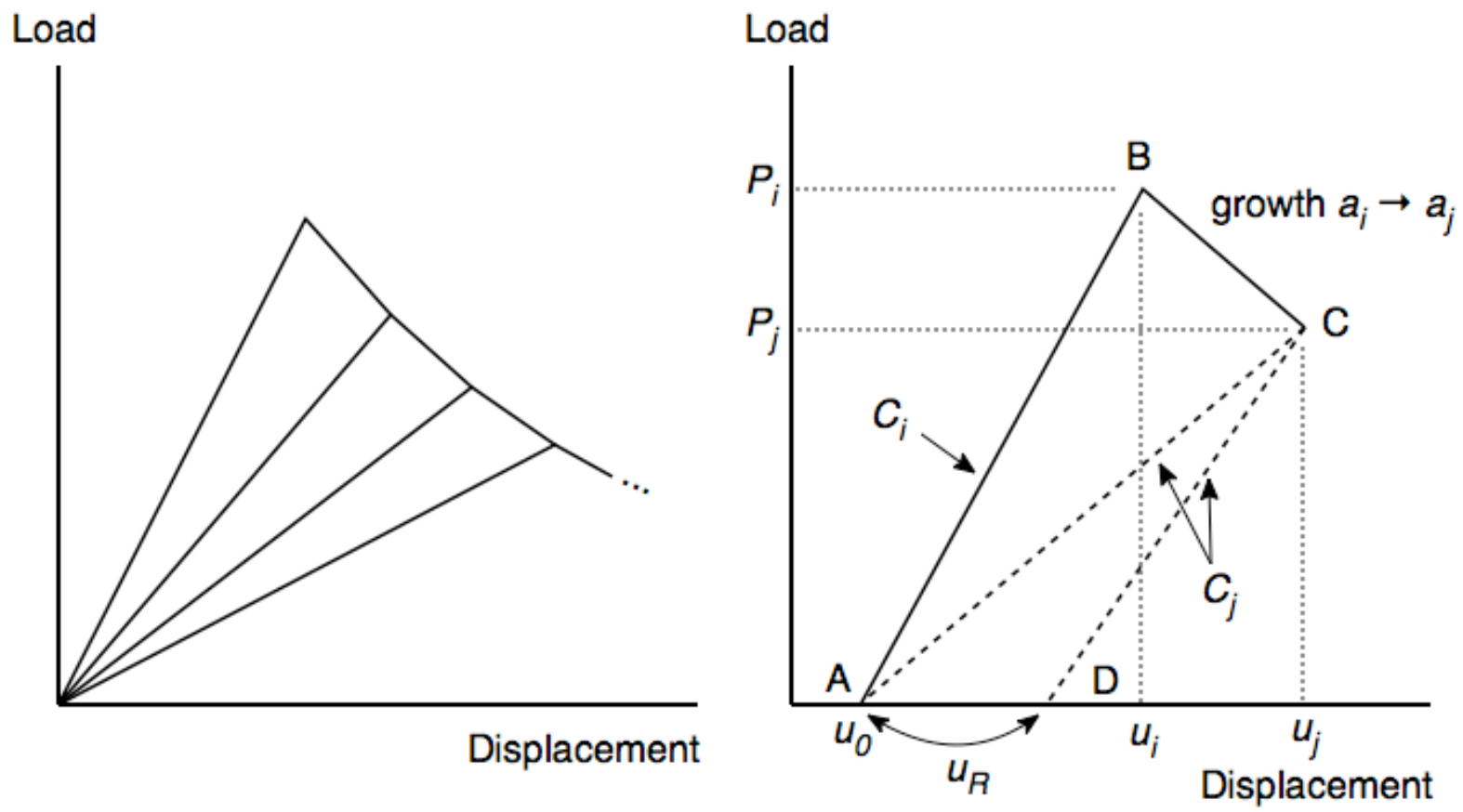

Figure 3: Left: load displacement curve for elastic fracture where the test is periodically stopped and unloaded. Right: A single loading and unloading envelop. Elastic fracture follows path ABC. Fracture with residual displacements follows path ABCD. 


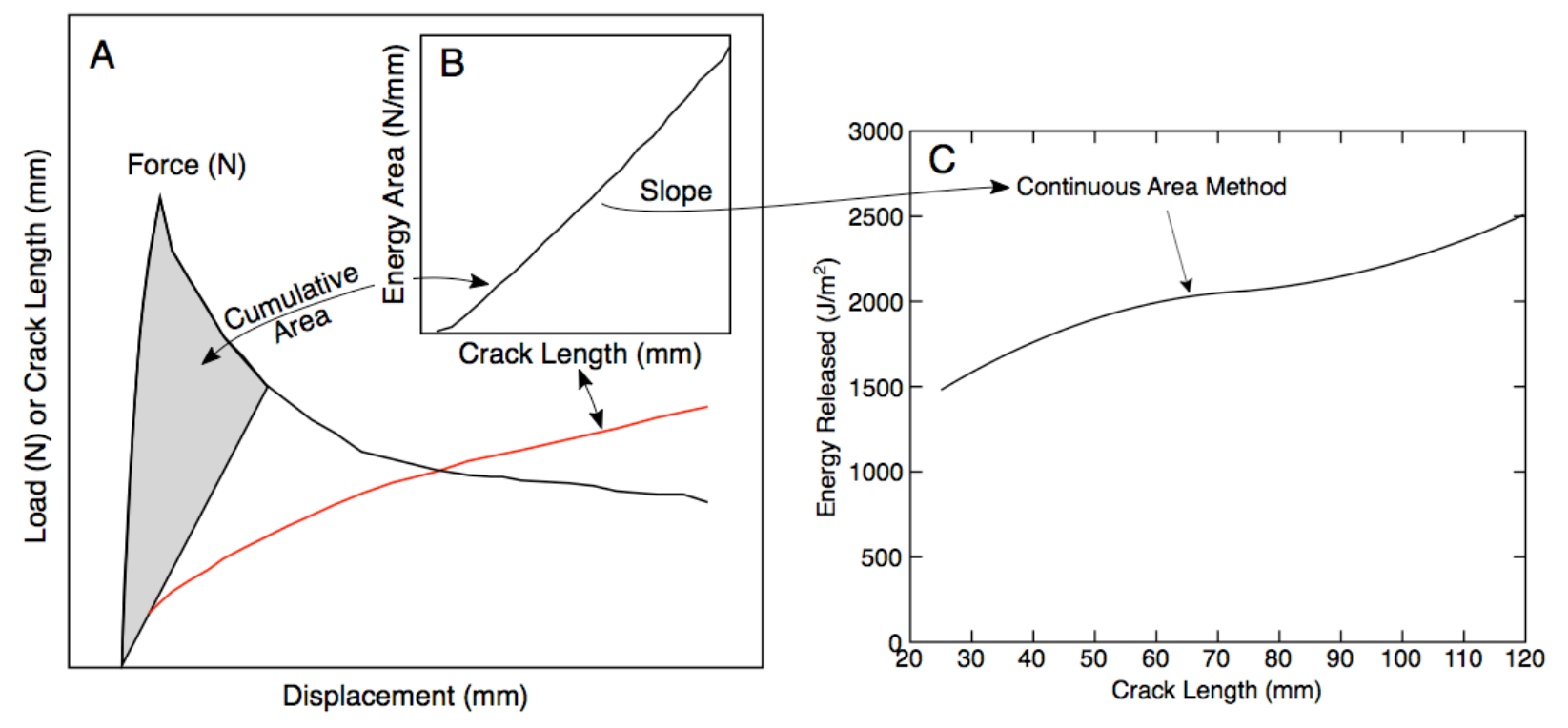

Figure 4: Graphical illustration of the revised $R$-curve method. The left shows integral transformation of force and crack length data as a function of displacement to cumulative energy as a function of crack length (B). C shows the $R$ curve as found from the slope of the energy area. 


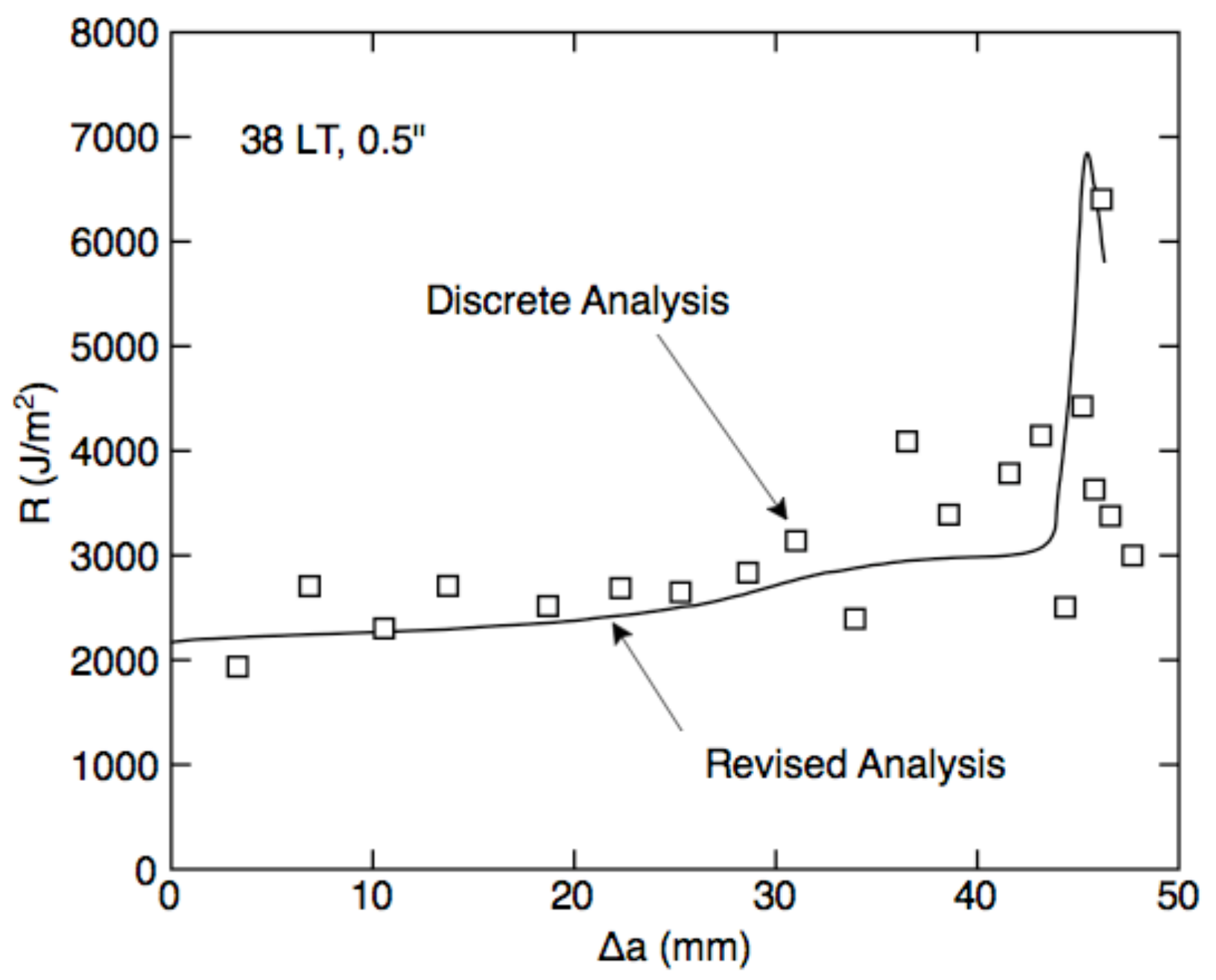

Figure 5: Analysis of the $R$ curve for $0.5 \mathrm{in}, 38 \mathrm{lb} / \mathrm{ft}^{3}$, LT fracture by two different methods. The symbols used the discrete method. The smooth line used the revised $R$-curve analysis method. 


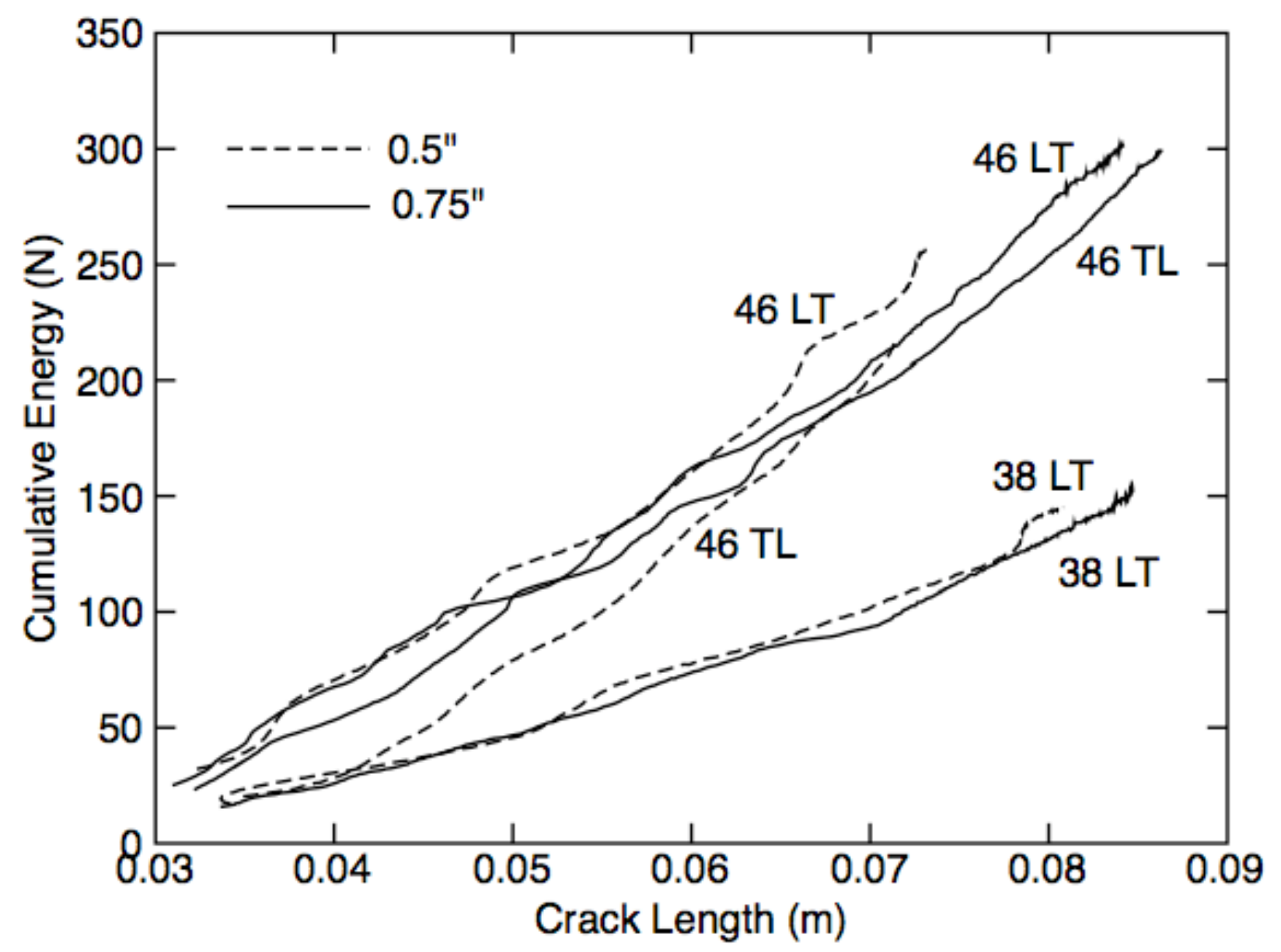

Figure 6: The cumulative energy released per unit thickness, $U(a)$, for all in-plane fracture specimens. The dashed lines are for 0.5 in thick panels; the solid lines are for 0.75 in thick panels. 


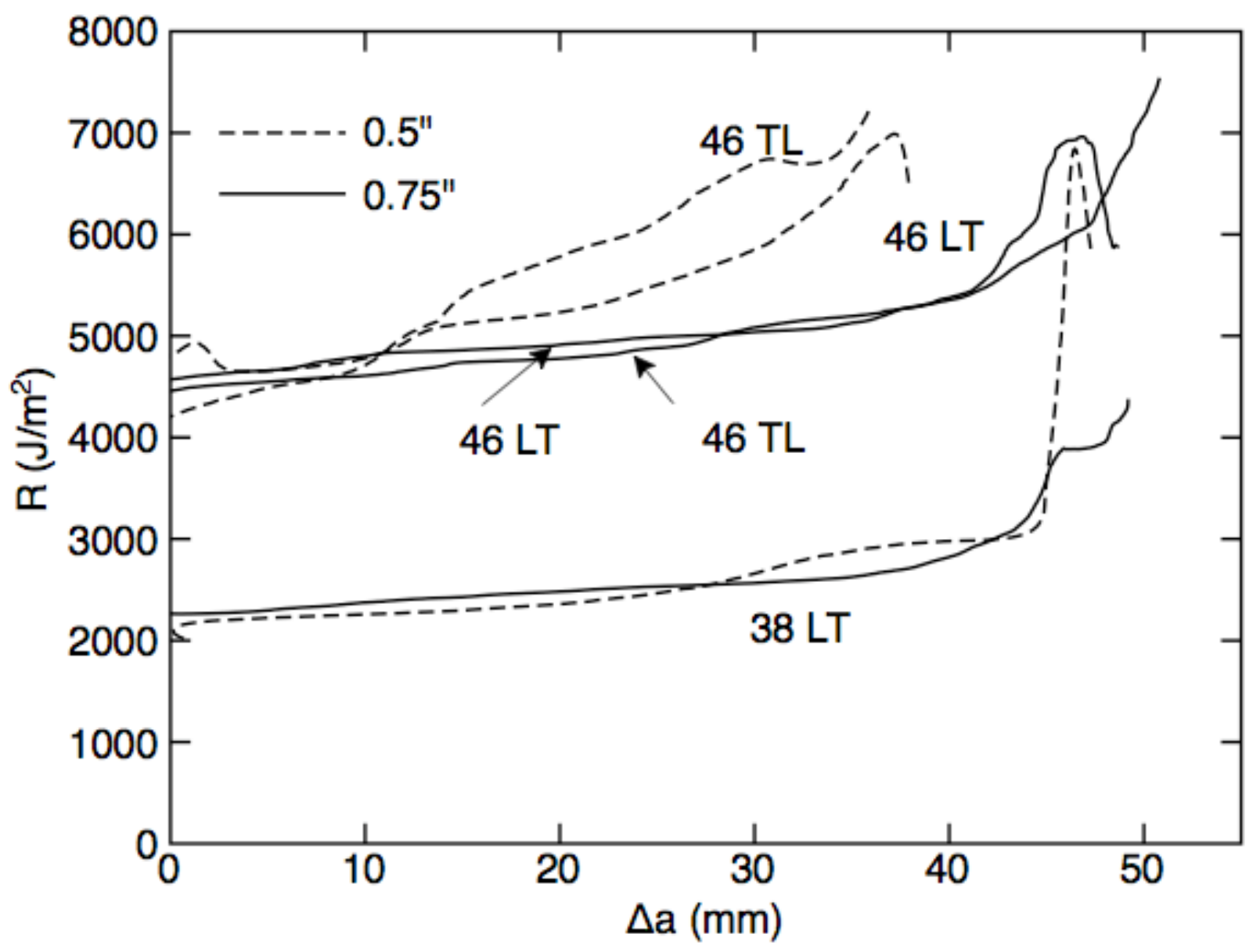

Figure 7: $R$ curves for all in-plane fracture experiments. The dashed lines are for 0.5 in thick panels; the solid lines are for 0.75 in thick panels. 


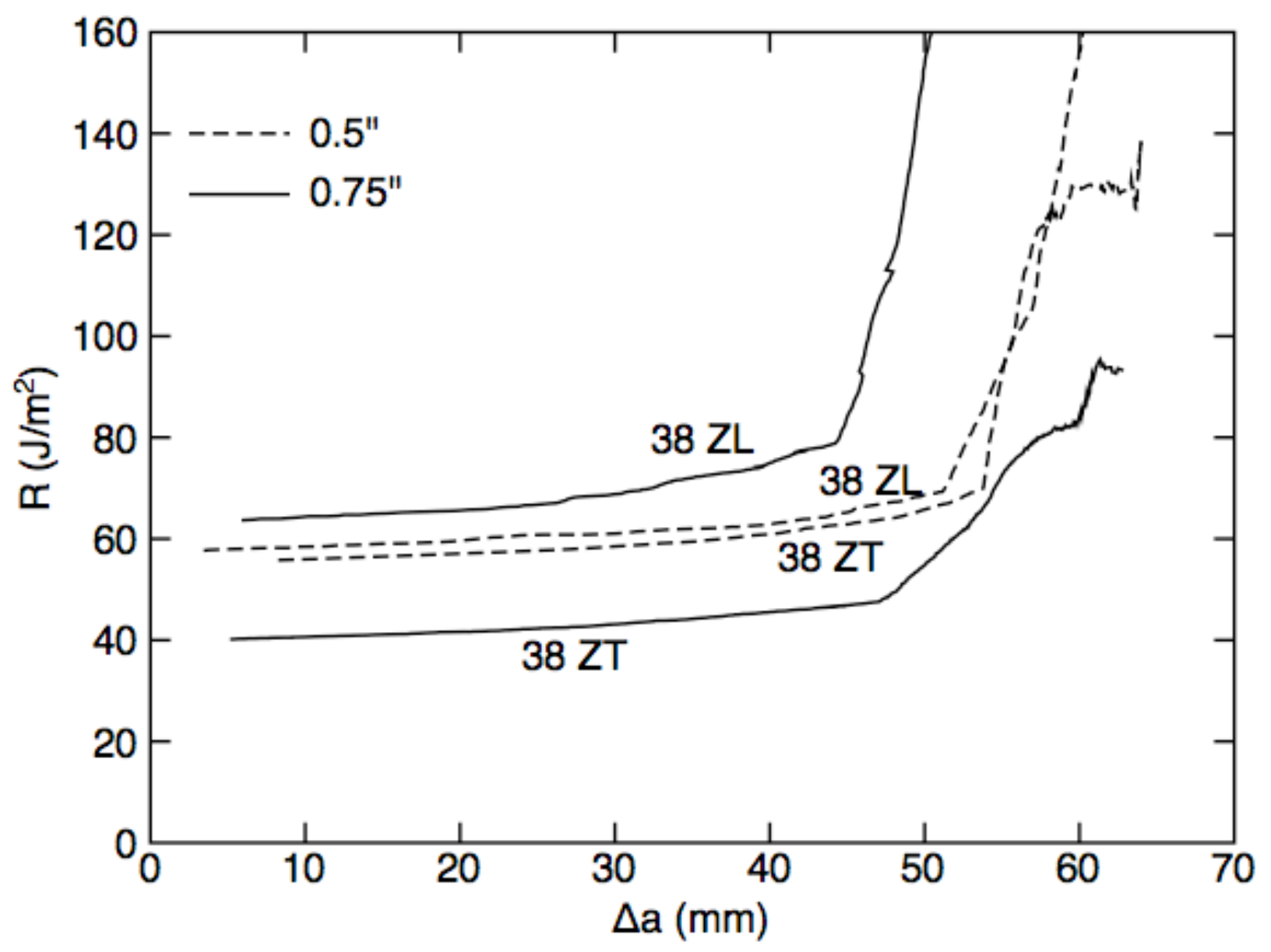

Figure 8: $R$ curves for all $Z$ cracks in the $38 \mathrm{lb} / \mathrm{ft}^{3}$ panels. The dashed lines are for 0.5 in thick panels; the solid lines are for 0.75 in thick panels. 


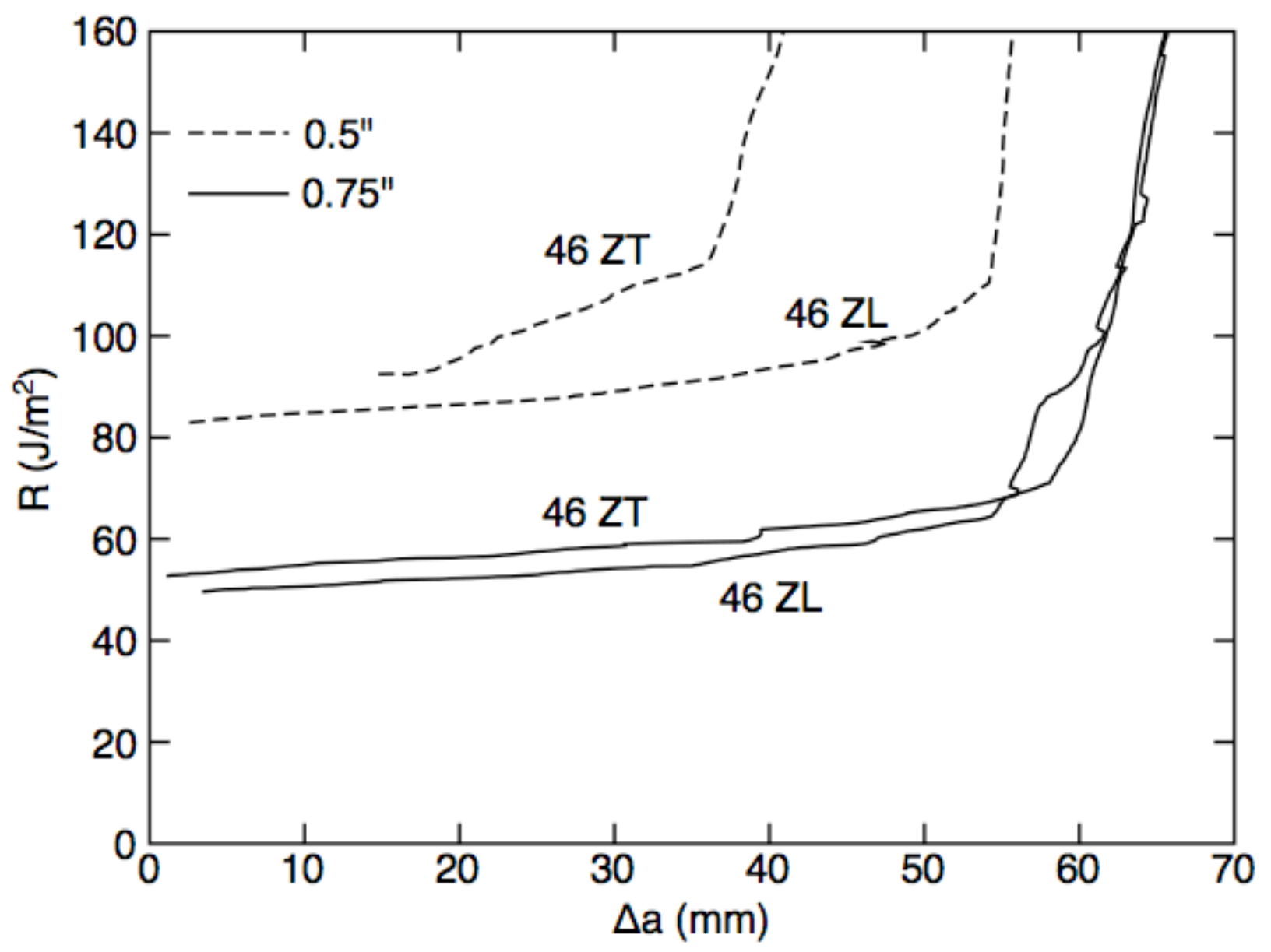

Figure 9: $R$ curves for all $Z$ cracks in the $46 \mathrm{lb} / \mathrm{ft}^{3}$ panels. The dashed lines are for 0.5 in thick panels; the solid lines are for 0.75 in thick panels. 

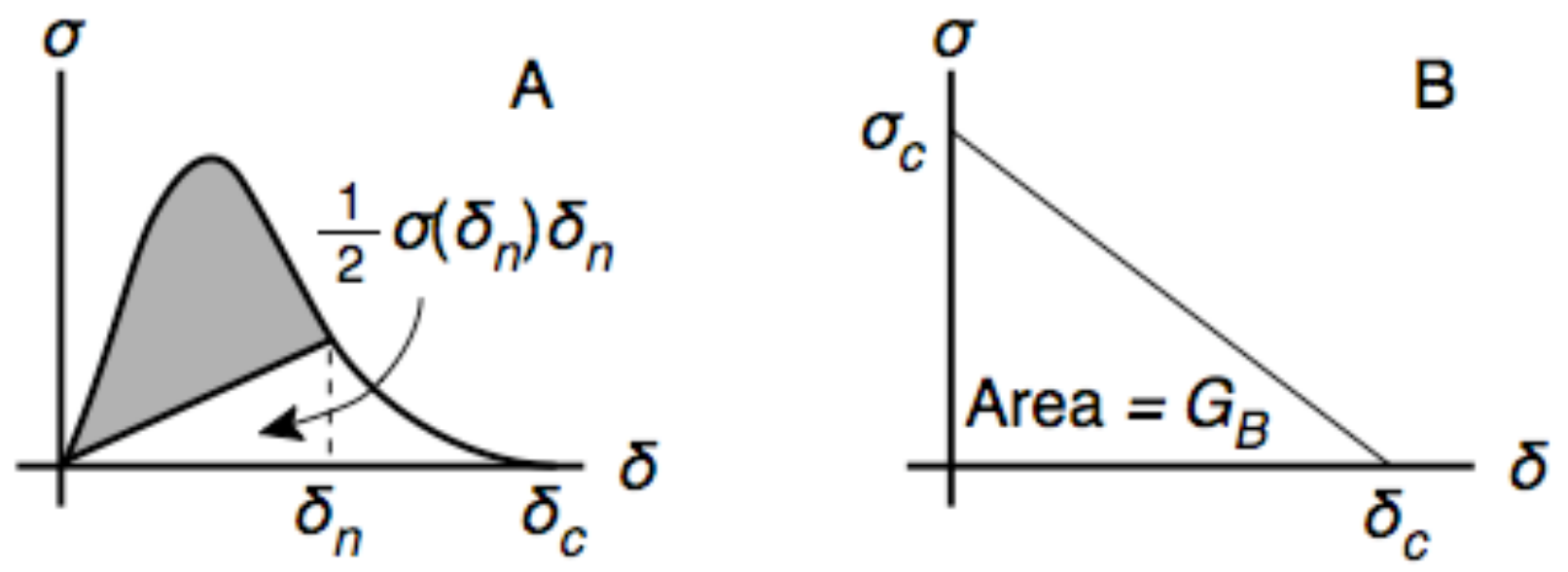

Figure 10: Fiber-bridging traction laws used to model the fiber bridging process zone. The shaded area in A illustrates the concept of energy released from the process zone prior to steady state crack propagation. B shows the linear softening law used to model fiber bridging in MDF. 


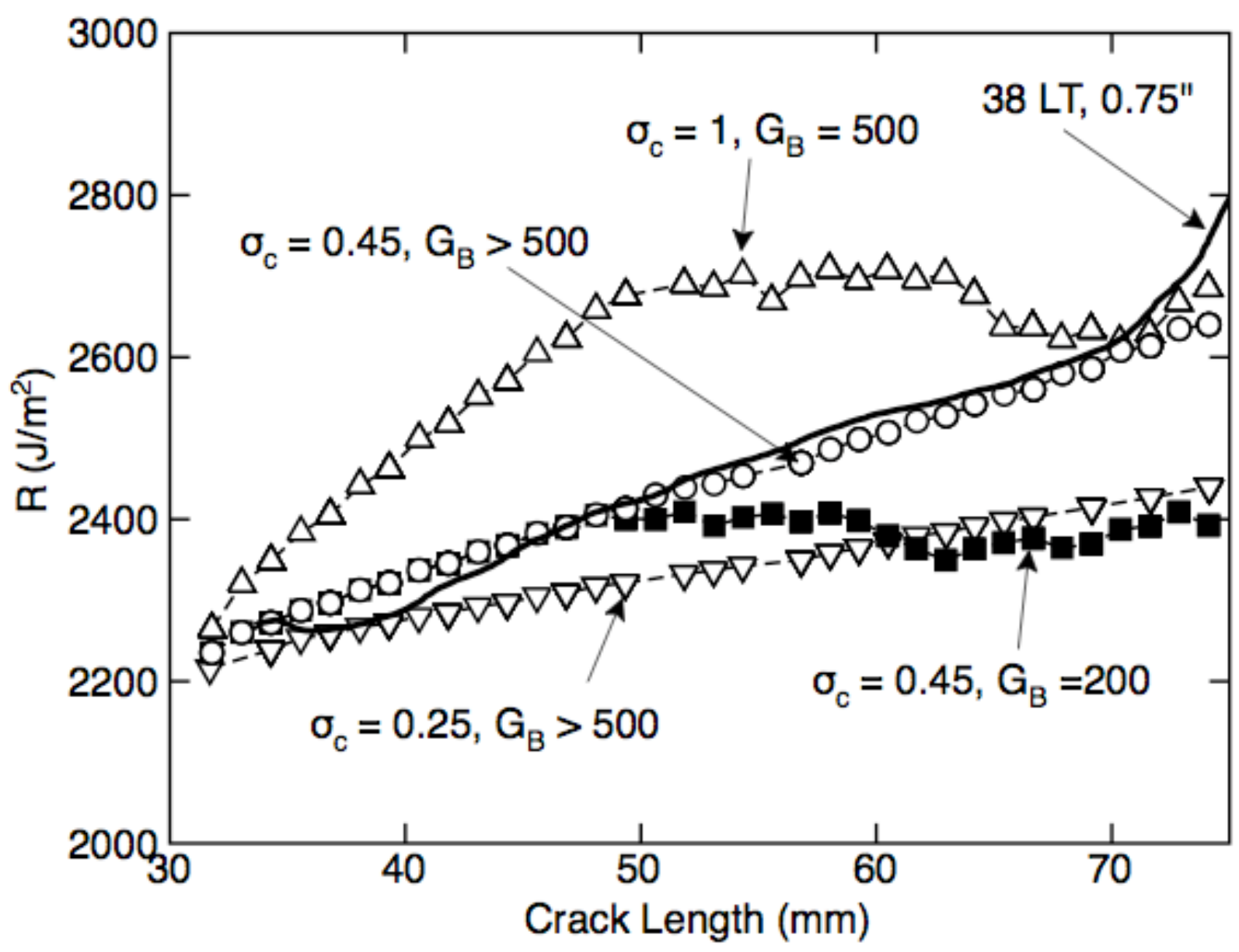

Figure 11: Comparison of simulation results (symbols) to experimental results (sold line) for LT crack growth in $0.75 \mathrm{in}, 38 \mathrm{lb} / \mathrm{ft}^{3}$ panels. The open symbols are for different values of $\sigma_{c}$ and $G_{B} \geq 500$. The solid symbols used a lower $G_{B}$. 